\title{
Isoquinolines from the Roots of Thalictrum flavum L. and Their Evaluation as Antiparasitic Compounds
}

\author{
Jacqueline Ropivia $^{1 \dagger}$, Séverine Derbré ${ }^{1, *}$, Caroline Rouger ${ }^{1}$, Fabrice Pagniez ${ }^{2}$, \\ Patrice Le Pape ${ }^{2}$ and Pascal Richomme ${ }^{1}$
}

$\dagger$ Dedicated to our friend Jacqueline Ropivia, in memoriam.

1 Laboratoire des Substances d'Origine Naturelle et Analogues Structuraux, UPRES-EA 921, IFR 149 QUASAV, UFR des Sciences pharmaceutiques et ingénierie de la santé, Université d'Angers, 16 Bd Daviers, 49045 Angers Cedex, France; E-Mail: pascal.richomme@univ-angers.fr (P.R.)

2 Laboratoire de Parasitologie et Mycologie médicale, BioCiT, UPRES EA 1155, Université de Nantes, Faculté de Pharmacie, 1 rue Gaston Veil, 44035 Nantes, France;

E-Mail: Patrice.Le-Pape@univ-nantes.fr (P.L.P.)

* Author to whom correspondence should be addressed; E-Mail: severine.derbre@univ-angers.fr; Tel.: +33 (0) 241226 663; Fax: +33 (0) 241226 634;

Received: 15 July 2010; in revised form: 24 August 2010 / Accepted: 13 September 2010 / Published: 16 September 2010

\begin{abstract}
Alkaloids from Thalictrum flavum L. (Ranuculaceae) growing in the Loire valley (France) were isolated and evaluated for their antiplasmodial and leishmanicidal activities. Berberine was identified as a major component but its analogue, pseudoberberine, was isolated for the first time from this plant. As far as bisbenzylisoquinolines are concerned, thalfoetidine was also isolated and, besides, its norderivative, northalfoetidine, was identified as a new compound. Previously isolated alkaloids from Thalictrum species such as northalidasine, northalrugosidine, thaligosidine, thalicberine, thaliglucinone, preocoteine, $O$-methylcassythine and armepavine were newly described in the roots of $T$. flavum. Tertiary isoquinolines, and particularly bisbenzylisoquinolines, were found to be leishmanicidal against L. major. Thalfoetidine appeared as the most potent but its new nor- derivative northalfoetidine, as well as northalidasine, were of particular interest due to the fact that their potential leishmanicidal activity was not associated to a strong cytotoxicity.
\end{abstract}


Keywords: Antiparasitic; bisbenzylisoquinolines; isoquinoline alkaloids; Ranunculaceae; Thalictrum flavum

\section{Introduction}

Widely distributed all over the world, Thalictrum species (Ranunculaceae) mainly grow in the temperate and cold zones of both hemispheres. Among secondary metabolites of these spp., isoquinoline alkaloids were the more investigated [1]. A variety of bisbenzylisoquinolines were isolated from roots and aerial parts of diverse Thalictrum [2]. These natural products are structurally and pharmacologically interesting since they exhibit specific antiparasitic properties [3], such as antimalarial [4,5] or antileishmanial activities [6-8]. Thalictrum flavum L., commonly known as "meadow-rue", is a 0.5 to $1.5 \mathrm{~m}$ height herb growing in tallgrass meadows, ditches, marshes and other habitats at the water's edge [9]. In spite of the fact that the alkaloid contents of T. flavum L. originating from Balkan countries [10-12] or Russia $[13,14]$ were already investigated, no pharmacological study has been reported so far.

Tropical parasitic diseases affect hundreds of millions of people, mostly in the Third World. As resistance to conventional treatments emerges, new drugs are still necessary in the treatment of protozoan parasitic diseases, such as malaria or leishmaniasis [15]. Natural products tend inherently to interact with living organisms and so are still a good source for compound leads [16]. In the present study, we describe the alkaloids of T. flavum L., together with their antiparasitic potential.

\section{Results and Discussion}

While complex and pharmacologically active dimeric alkaloids such as bisbenzylisoquinolines were already isolated from T. flavum L., structurally simpler isoquinolines were also extracted from this species [17]. The dimeric bisbenzylisoquinolines thalidasine, hernandesine and thalfoetidine were previously extracted from the roots as well from the aerial parts of this plant, whereas thalicarpine was only found in the roots and $O$-methylthalicberine only in the aerial parts. Glaucine and thalicsimidine (aporphines), thalicsine and thaliglucine (phenanthrenes), cryptopine (protopine) and thalflavine (isoquinolone) were also purified from the roots of $T$. flavum $[11,13,14]$. The oxoaporphines glaucine and corunnine were finally identified in the aerial parts of the plant [10,12]. Isoquinolines are structurally and pharmacologically interesting, since they exhibit specific antiparasitic properties [3] such as antimalarial [4,5] or antileishmanial activities [6-8]. However, no antiparasitic study for alkaloids from $T$. flavum $\mathrm{L}$. was previously reported.

In this work, as expected from a literature survey, all secondary metabolites isolated from T. flavum L. roots belong to the same isoquinoline group (Figure 1). As already described elsewhere, berberine (11) was identified as a major component $[11,13]$. However its analogue, pseudoberberine (12), was isolated for the first time from this plant. As far as bisbenzylisoquinolines are concerned, thalfoetidine (4) was also isolated in large amount from the roots [11] and, besides, its nor-derivative, northalfoetidine (5), was identified as a new compound. Previously isolated alkaloids from other Thalictrum species such as northalidasine (2), northalrugosidine (3), thaligosidine (6) and thalicberine 
(8) were characterized in the roots of $T$. flavum L. for the first time. It may be noticed that thalidasine was already isolated from underground parts [11] whereas $O$-methylthalicberine was described in aerial parts [10]. When phenanthrene derivatives were previously identified in the plant $[11,13]$, thaliglucinone (10) was here identified for the first time. Finally, the aporphines preocoteine (1) and $O$ methylcassythine (7), as well as the benzylisoquinoline armepavine (8), were newly described in this Thalictrum species.

Figure 1. Isoquinoline alkaloids isolated from Thalictrum flavum L. roots.

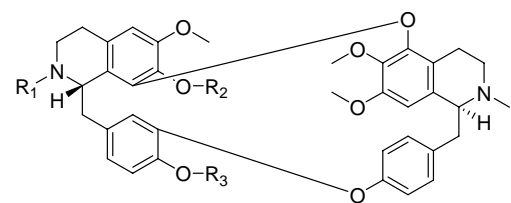

$\mathrm{R}_{1}=\mathrm{H}, \mathrm{R}_{2}=\mathrm{Me}, \mathrm{R}_{3}=$ Me: (-)-northalidasine 2 $\mathrm{R}_{1}=\mathrm{H}, \mathrm{R}_{2}=\mathrm{H}, \mathrm{R}_{3}=\mathrm{Me}:(-)$-northalrugosidine 3 $\mathrm{R}_{1}=\mathrm{Me}, \mathrm{R}_{2}=\mathrm{Me}, \mathrm{R}_{3}=\mathrm{H}:(-)$-thalfoetidine 4 $\mathrm{R}_{1}=\mathrm{H}, \mathrm{R}_{2}=\mathrm{Me}, \mathrm{R}_{3}=\mathrm{H}:(-)$-northalfoetidine 5

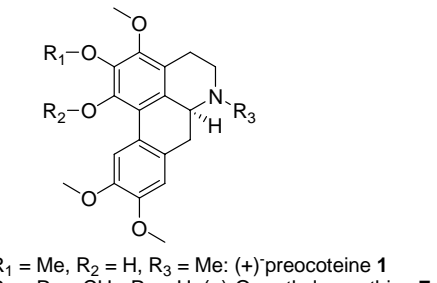

$\mathrm{R}_{1}=\mathrm{Me}, \mathrm{R}_{2}=\mathrm{H}, \mathrm{R}_{3}=\mathrm{Me:}(+)$-preocoteine 1
$\mathrm{R}_{1}=\mathrm{R}_{2}=\mathrm{CH}_{2}, \mathrm{R}_{3}=\mathrm{H}:(+)$-O-methylcassythine 7

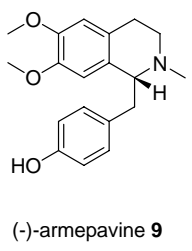

$(-)$-armepavine 9

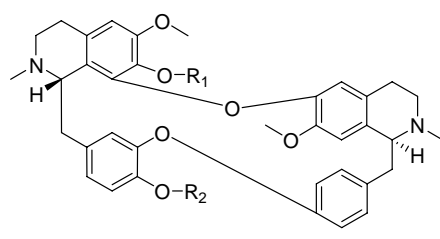

(+)-thalicberine 8
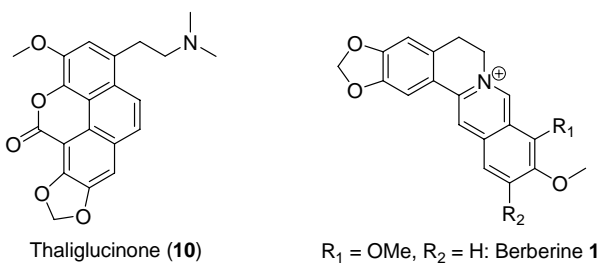

$\mathrm{R}_{1}=\mathrm{OMe}, \mathrm{R}_{2}=\mathrm{H}$ : Berberine $\mathbf{1 1}$ $\mathrm{R}_{1}=\mathrm{H}, \mathrm{R}_{2}=$ OMe: Pseudoberberine 12

Alkaloids isolated from T. flavum L. (Figure 1) were evaluated in assays evaluating their antiparasitic potential as well as their cytotoxicity. As depicted in Table 1, tertiary isoquinolines from $T$. flavum L. roots, and particularly bisbenzylisoquinolines, were found to be leishmanicidal against $L$. major. Thalfoetidine (4) appeared as the most potent but its new nor-derivative northalfoetidine (5), as well as northalidasine (2), were of particular interest due to the fact that their potential leishmanicidal activity was not associated to a strong cytotoxicity. As far as antiplasmodial activity was concerned, the aporphine preocoteine (1) and the bisbenzylisoquinoline thaligosidine (6) were more active but ten times less potent than the reference chloroquine. As expected, quaternary protoberberines $\mathbf{1 1}$ and 12 appeared as antiparasitic but cytotoxic [18].

\section{Experimental}

\subsection{General}

Silica gel 60 70-230 mesh (Merck 7754, Merck Chemicals, Fontenay-sous-Bois, France) and silica gel 60 230-400 mesh (Merck 9385) were used for column and flash chromatography respectively. Tlc analyses were performed on tlc silica gel 60 F254 (Merck) with Dragendorff reagent as developing agent.

${ }^{1} \mathrm{H}$ NMR, ${ }^{13} \mathrm{C}$ NMR and 2D NMR spectra were recorded in the appropriate deuterated solvent on a Bruker Avance DRX 500 MHz (Bruker France, Wissembourg, France) or a Jeol GSX $270 \mathrm{MHz}$ (Jeol Europe, Croissy-sur-Seine, France) spectrometer. Mass spectra were recorded on an Esquire 3000 
PLUS apparatus (Bruker). The ultraviolet spectra were obtained in $\mathrm{MeOH}$ on a Varian Cary 50 Bio UV-vis spectrometer (Varian France, Les Ulis, France). The infrared spectra were taken on a Bruker Vector 22 apparatus (Bruker), using chloroform as solvent.

\subsection{Plant material}

The roots of Thalictrum flavum L. were collected in May 2005 during flowering stage along the Loire river, near Angers (France). A voucher specimen was deposited in the Musée botanique d'Angers.

\subsection{Extraction and isolation}

Alkaloids of the air-dried and powdered roots of Thalictrum flavum L. were extracted following a classical protocol. Briefly, the plant material (300 g) was successively extracted with $\mathrm{CH}_{2} \mathrm{Cl}_{2} / \mathrm{MeOH}$ (1:1, $3 \mathrm{~L}), \mathrm{MeOH}(3 \mathrm{~L})$ and water (3 L). Organic layers were combined and evaporated under reduced pressure to yield $23.7 \mathrm{~g}$ of crude extract. This extract was solubilised in an acidic aqueous phase $(\mathrm{HCl}$ $0.1 \mathrm{~N}$ ) then successively washed with cyclohexane and $\mathrm{CH}_{2} \mathrm{Cl}_{2}$. The aqueous phase was subsequently alkalinised using $\mathrm{NH}_{4} \mathrm{OH}$, then extracted with $\mathrm{CHCl}_{3}$ to give $1.63 \mathrm{~g}$ of tertiary alkaloids.

This alkaloid extract (1.63 g) was subjected to a vacuum liquid chromatography (VLC) over silica gel using a gradient elution $\left(\mathrm{CH}_{2} \mathrm{Cl}_{2}\right.$ to $\mathrm{CH}_{2} \mathrm{Cl}_{2} / \mathrm{MeOH}$ (8:2) to yield 12 fractions (A-L).

Fraction $\mathrm{C}(58 \mathrm{mg})$ was purified using a medium pressure liquid chromatography (MPLC) over $\mathrm{Si}$ gel $\left[\mathrm{CHCl}_{3} / \mathrm{MeOH} / \mathrm{NH}_{4} \mathrm{OH}(95: 5: 0.2)\right]$ to give $6 \mathrm{mg}$ of (+)-preocoteine (1) [19,20].

Fraction E (191 mg) was subjected to MPLC over Si gel [hexane/EtOAc/DEA/MeOH (74:20:5:1)] to afford $7 \mathrm{mg}$ of (-)-northalidasine (2) [21], $7 \mathrm{mg}$ of (-)-northalrugosidine (3) [22], $29 \mathrm{mg}$ of (-)-thalfoetidine (4) [2,23] as well as $10 \mathrm{mg}$ of a new compound (5). Fractions 16-22 (20 mg) were subjected to preparative TLC using the same eluent to give $10 \mathrm{mg}$ of $4,4 \mathrm{mg}$ of (-)-thaligosidine (6) [24] and $2 \mathrm{mg}$ of (+)-O-methylcassythine (7) [25,26].

Fraction F (432 mg) was purified using a MPLC over Si gel [hexane/EtOAc/DEA/MeOH (73:20:5:2)] to give $11 \mathrm{mg}$ of $\mathbf{4}, 7 \mathrm{mg}$ of 5 and $53 \mathrm{mg}$ of (+)-thalicberine (8) [27]. Fractions F-9-15 $(80 \mathrm{mg})$ were also subjected to a MPLC, [cyclohexane/EtOAc/MeOH/DEA (90:5:5:5)] to give $3 \mathrm{mg}$ of 5, $16 \mathrm{mg}$ of 6 and $3 \mathrm{mg}$ of (-)-armepavine (9) [28]. Fractions F-20-34 (110 mg) were purified using MPLC with cyclohexane/EtOAc/MeOH/DEA (70:20:5:5) to give $24 \mathrm{mg}$ of (8) and $6 \mathrm{mg}$ of thaliglucinone (10) [29,30].

Fraction $\mathrm{H} \quad(208 \mathrm{mg})$ was finally purified through MPLC (Si gel) using cyclohexane $/ \mathrm{CH}_{2} \mathrm{Cl}_{2} / \mathrm{DEA} / \mathrm{MeOH}(70: 20: 5: 5)$ as the eluent to give $22 \mathrm{mg}$ of berberine (11) [18] and $51 \mathrm{mg}$ of pseudoberberine (12) [31,32]. 
Table 1. In vitro cytotoxicities and antiparasitic activities of compounds 1-12.

\begin{tabular}{|c|c|c|c|c|c|c|c|c|}
\hline \multirow{3}{*}{ Biological activities } & \multicolumn{4}{|c|}{ Cytotoxicity } & \multirow{2}{*}{\multicolumn{2}{|c|}{$\begin{array}{c}\text { Antimalarial } \mathrm{IC}_{50} \\
\text { Plasmodium falciparum } \\
\text { FcB1 Colombia }\end{array}$}} & \multirow{2}{*}{\multicolumn{2}{|c|}{$\begin{array}{l}\text { Leishmanicidal } \mathrm{IC}_{50} \\
\text { Leishmania major }\end{array}$}} \\
\hline & \multicolumn{2}{|c|}{ MCR-5 cells } & \multicolumn{2}{|c|}{$\mathrm{KB}$ cells } & & & & \\
\hline & $10 \mu \mathrm{g} / \mathrm{mL}$ & $1 \mu \mathrm{g} / \mathrm{mL}$ & $10 \mu \mathrm{g} / \mathrm{mL}$ & $1 \mu \mathrm{g} / \mathrm{mL}$ & $\mu \mathrm{g} / \mathrm{mL}$ & $\mu \mathrm{M}$ & $\mu \mathrm{g} / \mathrm{mL}$ & $\mu \mathrm{M}$ \\
\hline$(+)$-Preocoteine 1 & 20 & & 23 & & 0.5 & 1.3 & \multicolumn{2}{|c|}{$>100$} \\
\hline (+)-O-Methylcassythine 7 & 61 & 4 & 49 & & 3.1 & 8.6 & \multicolumn{2}{|c|}{ ND } \\
\hline (-)-Armepavine 9 & 0 & & 0 & & 3.6 & 11.4 & \multicolumn{2}{|c|}{ ND } \\
\hline (-)-Northalidasine 2 & 0 & & 32 & & 3.4 & 5.2 & 27 & 41 \\
\hline (-)-Northalrugosidine 3 & 77 & 0 & 51 & 0 & 2.7 & 4.3 & 30 & 48 \\
\hline (-)-Thalfoetidine 4 & 62 & 0 & 40 & & 2.1 & 3.3 & 17 & 27 \\
\hline (-)-Northalfoetidine 5 & 6 & & 44 & & 2.8 & 4.5 & 39 & 63 \\
\hline (-)-Thaligosidine 6 & 61 & 0 & 47 & & 1.2 & 2.0 & 38 & 61 \\
\hline (+)-Thalicberine 8 & 29 & & 43 & & 2.5 & 4.2 & 55 & 90 \\
\hline Thaliglucinone 10 & 79 & 21 & 76 & 24 & 2.4 & 6.7 & 63 & 173 \\
\hline Berberine 11 & ND & & ND & & 0.4 & 0.9 & 13 & 35 \\
\hline Pseudoberberine 12 & ND & & ND & & 0.5 & 1.3 & 3.5 & 9 \\
\hline Chloroquine & & & & & 0.06 & 0.1 & & \\
\hline Pentamidine & & & & & & & 28 & 82 \\
\hline
\end{tabular}


Compound 5 was identified as a new nor-derivative, (-)-northalfoetidine: Rf 0.35 (hexane/EtOAc/DEA/MeOH 70:20:5:5). UV (MeOH) $\lambda \max (\mathrm{nm}): 275 .{ }^{1} \mathrm{H}-\mathrm{NMR}\left(500 \mathrm{MHz}, \mathrm{CDCl}_{3}\right.$ ): 7.52 (1 H, dd, $J=8.5,2$ Hz, H-14'), 6.97 (1 H, dd, $J=8.5,2$ Hz, H-13'), 6.81 (2 H, d, $J=9$ Hz, H-13, H-14), 6.48 (1 H, dd, $J=8.5,2$ Hz, H-11'), 6.45 (1 H, s, H-8'), 6.34 (1 H, dd, J = 8.5, 2 Hz, H-10'), 6.30 (1 H, s, H-5), 6.28 (1 H, d, J=2 Hz, H-10), 3.89 (2 H, m, H-1, H-1'), 3.87 (3 H, s, 6'-OMe), 3.76 (3 H, s, 6-OMe), 3.47 (3 H, s, 7-OMe), 3.27 (3 H, s, 7'-OMe), 3.23 (2 H, m, H- $\alpha, \mathrm{H}-\alpha^{\prime}$ ), 2.90 (2 H, m, H-3, H- 3'), 2.80 (2 H, m, H- $\left.\alpha, H_{-} \alpha^{\prime}\right), 2.65$ (3 H, s, N'-Me), 2.40 (2 H, m, H-4, H- 4'), 2.35 (2 H, $m$, H-3, H- 3'), 2.15 (2 H, m, H-4, H- 4'). ${ }^{13} \mathrm{C}-\mathrm{NMR}$ (125 MHz, $\left.\mathrm{CDCl}_{3}\right)$ : 153.5 (C-12'), 152.0 (C-6), 151.0 (C-6'), 147.7 (C-11 or C-12), 147.1 (C-11 or C-12), 143.8 (C-8, C-5'), 137.8 (C-7), 137.6 (C-7'), 134.2 (C-9, C-9'), 132.5 (C-14'), 132.2 (C-4a'), 131.2 (C-10'), 124.0 (C-8a), 121.6 (C-4a), 119.4 (C13'), 119.2 (C-11'), 114.9 (C-13, C-14), 114.3 (C-10), 106.1 (C-8'), 105.4 (C-5), 64.9 (C-1, C-1'), 60.5 (7'-OMe), 60.2 (7-OMe), 56.2 (6'-OMe), 55.8 (6-OMe), 51.2 (C-3, C-3'), 43.9 (N'-Me), 40.8 (C$\left.\alpha, \mathrm{C}^{\prime} \alpha^{\prime}\right), 24.1\left(\mathrm{C}-4, \mathrm{C}-4\right.$ '). HREIMS: $m / z 625.2908$ (calcd for $\left.\mathrm{C}_{37} \mathrm{H}_{41} \mathrm{~N}_{2} \mathrm{O}_{7}{ }^{+}, 625.2909\right)$. $[\alpha]_{\mathrm{D}}{ }^{20^{\circ} \mathrm{C}}$ : - $27(\mathrm{c}$ $\left.=0.07, \mathrm{CHCl}_{3}\right)$.

\subsection{Evaluations of cytotoxicity towards MCR-5 and KB cells}

Cytotoxicities were evaluated at the ICSN-CNRS (Gif sur Yvette, France) on MRC5 and KB cells, in DMSO, at 10 and $1 \mu \mathrm{g} / \mathrm{mL}$ according to the procedure described by Moret et al. [33].

\subsection{In vitro $P$. falciparum culture and activity assays}

Antimalarial activity was evaluated on FcB1 Colombia strain of Plasmodium falciparum according to a previously described procedure [34].

\subsection{In vitro leishmanicidal activity}

Leishmania major (MHOM/Il/81/BNI) was cultivated at $26{ }^{\circ} \mathrm{C}$ in a Schneider's insect medium (Sigma, St Quentin Fallavier, France) supplemented with 15\% foetal bovine serum (FBS) (Sigma), penicillin $(100 \mathrm{IU} / \mathrm{mL})$ and streptomycin $(50 \mu \mathrm{g} / \mathrm{mL})$. Exponentially growing cells were maintained at $26{ }^{\circ} \mathrm{C}$. Promastigote susceptibility testing was performed with the Uptiblue ${ }^{\circledR}$ micromethod previously described [35]. Briefly, $100 \mu \mathrm{L}$ of a 106 promastigotes $/ \mathrm{mL}$ suspension were placed into wells of a 96well microplate (Nunc $\left.{ }^{\circledR}\right)$. The cultures were exposed for $96 \mathrm{~h}$ at $26^{\circ} \mathrm{C}$ to the antileishmanial drugs at the concentrations used above, except for meglumine antimoniate for which dilutions were $0.2,2$ and $20 \mathrm{mg} \cdot \mathrm{mL}^{-1}$. Four $\mathrm{h}$ before measurement, $10 \mu \mathrm{L}$ of Uptiblue ${ }^{\circledR}$ were added. The fluorescence was measured at $590 \mathrm{~nm}$ with an excitation at $550 \mathrm{~nm}$.

\section{Conclusion}

In summary, twelve isoquinolines were isolated from the roots of Thalictrum flavum L. As expected, berberine $\mathbf{1 1}$ was identified as the major component but its analogue, pseudoberberine 12, was isolated for the first time from this plant. Six bisbenzylisoquinolines (2-6 and 8) were identified: As previously described, thalfoetidine 4 was isolated in large amount but, more interesting, its nor- 
derivative, northalfoetidine 5, was identified as a new compound. Moreover, bisbenzylisoquinoline, aporphine, phenanthrene and benzylisoquinoline alkaloids, previously isolated from Thalictrum species, were newly described in the roots of $T$. flavum. This chemical composition is slightly different from those previously described. This could be due to either seasonal variations or a difference in the soil type. Indeed, previous studies were on T. flavum from Balkan countries or Russia, while we worked on a sample originating from the Loire valley. As far as antiparasitic activity was concerned, tertiary isoquinolines, especially bisbenzylisoquinolines, were found to be leishmanicidal against $L$. major. Thalfoetidine $\mathbf{4}$ appeared as the most potent but its nor- derivative northalfoetidine $\mathbf{5}$, as well as northalidasine 2 , were of particular interest as their potential leishmanicidal activity was not associated to a strong cytotoxicity.

\section{Acknowledgements}

The authors swish to thank Geneviève Aubert ICSN-CNRS (Gif sur Yvette, France) for performing the cytotoxic assays. Philippe Grellier from the Muséum National d'Histoire Naturelle, (Paris, France) is also thanked for antiplasmodial assays.

\section{References}

1. Khamidullina, E.A.; Gromova, A.S.; Lutsky, V.I.; Owen, N.L. Natural products from medicinal plants: non-alkaloidal natural constituents of the Thalictrum species. Nat. Prod. Rep. 2006, 23, 117-129.

2. Guha, K.P.; Mukherjee, B.; Mukherjee, R. Bisbenzylisoquinoline alkaloids -A review. J. Nat. Prod. 1979, 42, 1-84.

3. Kayser, O.; Kiderlen, A.F.; Croft, S.L. Natural products as antiparasitic drugs. Parasitol. Res. 2003, 90, S55-S62.

4. Angerhofer, C.K.; Guinaudeau, H.; Wongpanich, V.; Pezzuto, J.M.; Cordell, G.A. Antiplasmodial and cytotoxic activity of natural bisbenzylisoquinoline alkaloids. J. Nat. Prod. 1999, 62, 59-66.

5. Kaur, K.; Jain, M.; Kaur, T.; Jain, R. Antimalarials from nature. Bioorg. Med. Chem. 2009, 17, 3229-3256.

6. Fournet, A.; Muñoz, V.; Manjon, A.M.; Angelo, A.; Hocquemiller, R.; Cortes, D.; Cave, A.; Bruneton, J. Activité antiparasitaire d'alcaloïdes bisbenzylisoquinoleiques. I: activité in vitro sur des promastigotes de trois souches de Leishmania. J. Ethnopharmacol. 1988, 24, 327-335.

7. Maria del Rayo, C.; Phillipson, J.D.; Simon, L.C.; Peter, R.; Sarah, J.M.; Paul, L.S., Jr. In vitro activity of Triclisia patens and some bisbenzylisoquinoline alkaloids against Leishmania donovani and Trypanosoma brucei brucei. Phytother. Res. 2002, 16, 432-436.

8. Mishra, B.B.; Singh, R.K.; Srivastava, A.; Tripathi, V.J.; Tiwari, V.K. Fighting against leishmaniasis: Search of alkaloids as future true potential anti-leishmanial agents. Mini-Rev. Med. Chem. 2009, 9, 107-123.

9. Bonnier, G. La grande flore en couleurs de Gaston Bonnier. France, Suisse, Belgique et pays voisins; Editions Belin: Paris, France, 1990; p. 1401.

10. Dutschewska, H.; Velcheva, M.; Samuelsson, G. The alkaloids of aerial parts of Thalictrum flavum L. Acta Pharm. Nordica 1989, 1, 363-366. 
11. Velcheva, M.; Dutschewska, H.; Samuelsson, G. The alkaloids of the roots of Thalictrum flavum L. Acta Pharm. Nordica 1992, 4, 57-58.

12. Popović, M.; Djurković, R.; Gašić, O.; Boža, P.; Dutschevska, H.; Kuzmanov, B. Chemical and cytological investigation of Thalictrum flavum L. from the Vojvodina region. J. Serb. Chem. Soc. 1996, 61, 159-163.

13. Ismailov, Z.; Lutfullin, K.; Yunusov, S. Alkaloids of the roots of Thalictrum flavum. Chem. Nat. Compd. 1968, 4, 173-174.

14. Umarov, K.; Ismailov, Z.; Yunusov, S. Alkaloids of Thalictrum flavum. Chem. Nat. Compd. 1970, 6, 452-454.

15. Renslo, A.R.; McKerrow, J.H. Drug discovery and development for neglected parasitic diseases. Nat. Chem. Biol. 2006, 2, 701-710.

16. Newman, D.J.; Cragg, G.M. Natural products as sources of new drugs over the last 25 years. J. Nat. Prod. 2007, 70, 461-477.

17. Schiff, P.L. Alkaloids: Chemical and Biological Perspectives, 1st ed.; Pelletier, S.W., Ed.; Pergamon: Oxford, UK, 1996; Volume 11, pp. 1-236.

18. Grycová, L.; Dostál, J.; Marek, R. Quaternary protoberberine alkaloids. Phytochemistry 2007, 68, 150-175.

19. Guinaudeau, H.; Leboeuf, M.; Cavé, A. Aporphinoid alkaloids IV. J. Nat. Prod. 1988, 51, 389-474.

20. Nimgirawath, S.; Udomputtimekakul, P.; Taechowisan, T.; Wanbanjob, A.; Shen, Y. First total syntheses of $( \pm$ )-isopiline, $( \pm)$-preocoteine, $( \pm)$-oureguattidine and $( \pm)$-3-methoxynordomesticine and the biological activities of ( \pm -3-methoxynordomesticine. Chem. Pharm. Bull. 2009, 57, 368-376.

21. Hussain, S.F.; Guinaudeau, H.; Freyer, A.J.; Shamma, M. Bisbenzylisoquinoline alkaloids from Thalictrum cultratum. The structures of thalrugosinone and thalpindione. J. Nat. Prod. 1985, 48, 962-966.

22. Wu, W.-N.; Beal, J.L.; Doskotch, R.W. Alkaloids of Thalictrum. XXXIII. Isolation and characterization of alkaloids from the root of Thalictrum alpinum. J. Nat. Prod. 1980, 43, 372-381.

23. Georgiev, V.S.; Mollov, N.M. The structure of thalfoetidine. Phytochemistry 1971, 10, 2161-2166.

24. Wu, W.-N.; Beal, J.L.; Fairchild, E.H.; Doskotch, R.W. Alkaloids of Thalictrum. 25. Six new bisbenzylisoquinoline alkaloids from Thalictrum rugosum. J. Org. Chem. 1978, 43, 580-585.

25. Jones, S.R.; Lamberton, J.A. Cassytha alkaloids. I. New aporphine alkaloids from Cassytha filiformis L. Aust. J. Chem. 1966, 19, 297-302.

26. Tsai, T.-H.; Wang, G.-J.; Lin, L.-C. Vasorelaxing alkaloids and flavonoids from Cassytha filiformis. J. Nat. Prod. 2008, 71, 289-291.

27. Guinaudeau, H.; Freyer, A.J.; Shamma, M. Spectral characteristics of the bisbenzylisoquinoline alkaloids. Nat. Prod. Rep. 1986, 3, 477-488.

28. Atta-ur-Rahman. Handbook of Natural Products Data. Isoquinoline Alkaloids; Elsevier: Amsterdam, The Netherlands, 1994; Volume 3, p. 774. 
29. Shamma, M.; Moniot, J.L.; Yao, S.Y.; Stanko, J.A. Two isoquinoline alkaloids with a methylenoxy-bridge. J. Chem. Soc., Chem. Commun. 1972, 408-409.

30. Brossi, A.; Manske, R.H.F.; Holmes, H.L. The Alkaloids: Chemistry and Pharmacology; Academic Press Inc: San Diego, CA, USA, 1990; Volume 39, p. 364.

31. Moulis, C.; Gleye, J.; Stanislas, E. Alcaloïdes de l' Isopyrum thalictroïdes - bases quaternaires des feuilles - isolement et identification de quatre pseudoprotoberberines. Phytochemistry 1977, 16, 1283-1287.

32. Saied, S.; Batool, S.; Naz, S. Phytochemical studies of Berberis aristata. J. Basic Appl. Sci. 2007, 3, 1-4.

33. Moret, V.; Laras, Y.; Cresteil, T.; Aubert, G.; Ping, D.Q.; Di, C.; Barthélémy-Requin, M.; Béclin, C.; Peyrot, V.; Allegro, D.; Rolland, A.; De Angelis, F.; Gatti, E.; Pierre, P.; Pasquini, L.; Petrucci, E.; Testa, U.; Kraus, J.-L. Discovery of a new family of bis-8-hydroxyquinoline substituted benzylamines with pro-apoptotic activity in cancer cells: Synthesis, structure-activity relationship, and action mechanism studies. Eur. J. Med. Chem. 2009, 44, 558-567.

34. Krief, S.; Martin, M.-T.; Grellier, P.; Kasenene, J.; Sevenet, T. Novel antimalarial compounds isolated in a survey of self-medicative behavior of wild chimpanzees in Uganda. Antimicrob. Agents Chemother. 2004, 48, 3196-3199.

35. Le Pape, P.; Pagniez, F.; Abdala, H. A new fluorometric method for anti-Leishmania drug screening on axenic amastigotes. Acta Parasitol. 2003, 48, 41-79.

Sample Availability: Samples of compounds 1 to 12 are available from the authors.

(C) 2010 by the authors; licensee MDPI, Basel, Switzerland. This article is an open access article distributed under the terms and conditions of the Creative Commons Attribution license (http://creativecommons.org/licenses/by/3.0/). 